\title{
Mineral 4/Recognition 4: A Universal Optical Image Analysis Package for Iron Ore, Sinter and Coke Characterization
}

\author{
Eugene Donskoi ${ }^{1}$, Andrei Poliakov ${ }^{2}$, Keith Vining $^{3}$ and Sarath Hapugoda ${ }^{4}$ \\ 1. Project Leader, Mineral Processing Modelling, CSIRO Mineral Resources, PO Box 883, Kenmore QLD 4069, Australia \\ 2. Senior Experimental Scientist, CSIRO Mineral Resources, PO Box 883, Kenmore QLD 4069, Australia \\ 3. Group Leader - Carbon Steel Futures, CSIRO Mineral Resources, PO Box 883, Kenmore QLD 4069, Australia \\ 4. Process Mineralogist, CSIRO Mineral Resources, PO Box 883, Kenmore QLD 4069, Australia
}

Received: September 23, 2016 / Accepted: October 11, 2016 / Published: January 31, 2017.

\begin{abstract}
OIA (optical image analysis) has traditionally been used for reliable identification of different iron oxides and oxyhydroxides in iron ore. The automated CSIRO OIA system Mineral 4/Recognition 4 was created for rapid mineral and textural characterisation of iron ore providing identification of different minerals and different morphologies. The technique has further been applied to processed iron ore products such as iron ore sinter to determine key parameters such as porosity, different morphologies of hematite (primary and secondary), and different morphologies of SFCA (silicon ferrite of calcium and aluminium). Application of textural identification has recently been extended to coke characterisation where the software gives comprehensive characterisation of porosity, IMDC (inert material derived components), RMDC (reactive material derived components) and the boundaries between IMDC and RMDC. The software also has many unique features needed for iron ore research including characterisation of large objects like pellets and ore lumps; automated gangue (including quartz) identification; automated particle separation; multiple image set processing and on-line measurements. All these features make the Mineral 4/Recognition 4 OIA system a unique, reliable, industry/research focused tool for ore, sinter, pellet and coke characterisation.
\end{abstract}

Key words: Image analysis, automated, characterisation, iron ore, sinter, coke.

\section{Introduction}

The development of new complex iron ore deposits together with the need to maximise iron grade and recovery from beneficiation processes and to optimise agglomeration processes demands a robust technique for the comprehensive characterisation of iron ores and its processed products.

Typically, chemical and mineral composition is determined by XRF (X-ray fluorescence) and XRD (X-ray diffraction) analysis, respectively. However, information about mineral liberation and associations

Corresponding author: Eugene Donskoi, Ph.D., project leader, research fields: mineral characterisation and mineral processing modelling. is often required as this will affect subsequent beneficiation processes. These are commonly obtained from two or three dimensional imaging techniques [1, 2]. Ores with similar mineralogy can behave differently during processing, as the behaviour is also determined by the spatial distribution of different minerals and porosity (that is, textural composition) of the ore [3, 4]. As an example of the differences in texture possible for a chemically simple mineral such as hematite, Fig. 1 shows four different particles that consist primarily of hematite, but exhibit significantly different textures. These different textures have different characteristics in terms of hardness, attrition resistance, moisture absorption, and will behave differently during comminution and beneficiation processes as well as 


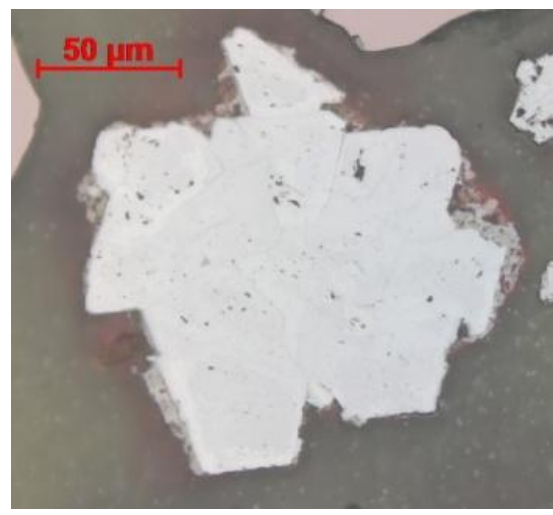

(a)

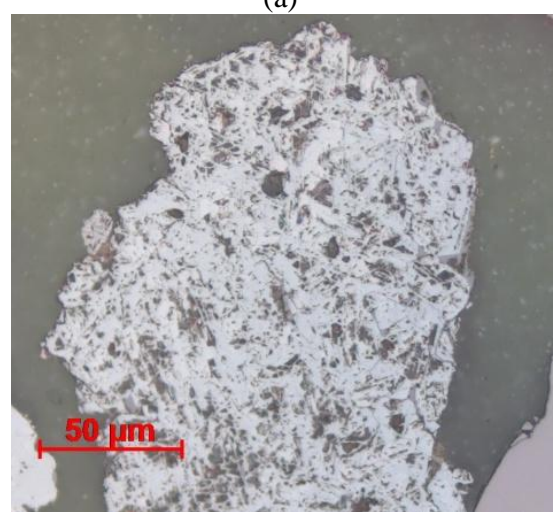

(c)

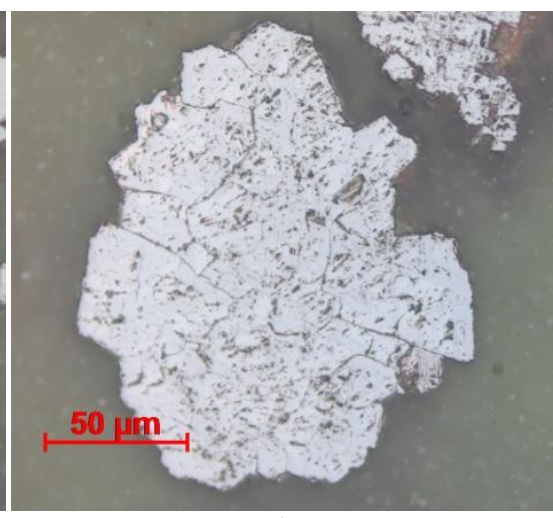

(b)

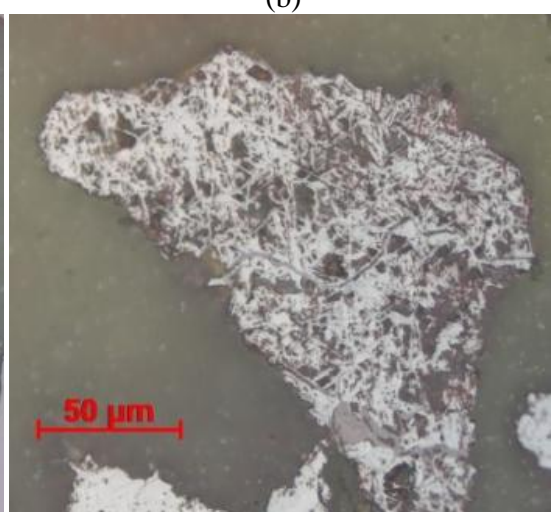

(d)

Fig. 1 Examples of four hematitic particles exhibiting different textural types ranging from dense (a) to highly porous (d).

granulation and sintering. Ideally each of these examples should be attributed to different textural classes.

Particles with similar textures are likely to express similar behaviour during downstream processing. Consequently, characterising ore as a combination of certain textures, together with the known response of each textural type to certain processing conditions, would improve prediction of the ore overall behaviour during processing.

Two major imaging techniques are currently used to characterise iron making materials: SEM (scanning electron microscopy) [5-7] and OIA (optical image analysis) [8-11]. Both techniques have their advantages and drawbacks. SEM methods better characterise gangue including non-opaque minerals and give simultaneous chemical composition, while OIA is more cost effective, better distinguishes between different iron oxides and oxyhydroxides (see Fig. 2 for mineral segmentation by Mineral 4, reproduced from Ref. [9]), has better resolution for massive screening and better characterises porosity (for detailed comparison of the two techniques see Refs. [3, 12]).

This article briefly describes the automated CSIRO optical image analysis system Mineral 4/Recognition 4 before showing examples of the application of the software to the characterisation of materials used and produced in iron making. We provide examples of the application of the method to iron ore and coke feed materials as well as to processed products such as iron ore sinter and pellets.

\section{Importance of Textural Classification}

Recognition of the importance of textural information for the prediction of downstream processing is steadily increasing among the industry and academics [3, 13].

Fig. 3 is an example from mathematical modelling of sinter cold strength (TI (Tumble Index)) as a performance indicator of sintering performance of iron ore fines [14, 15]. Fig. 3 (reproduced from Ref. [15]) 


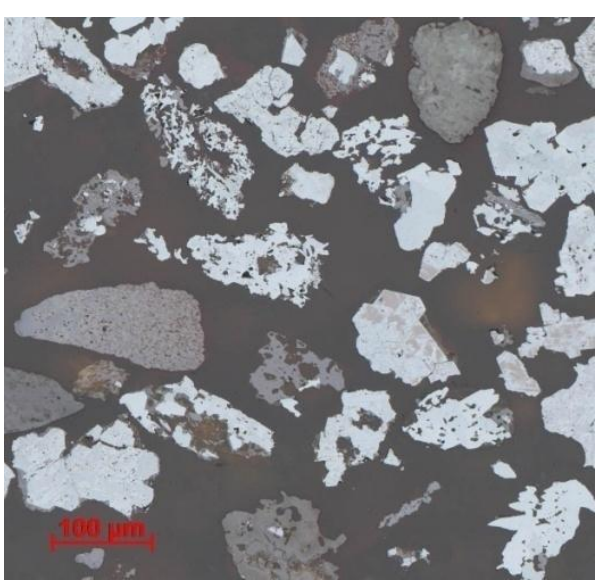

(a)

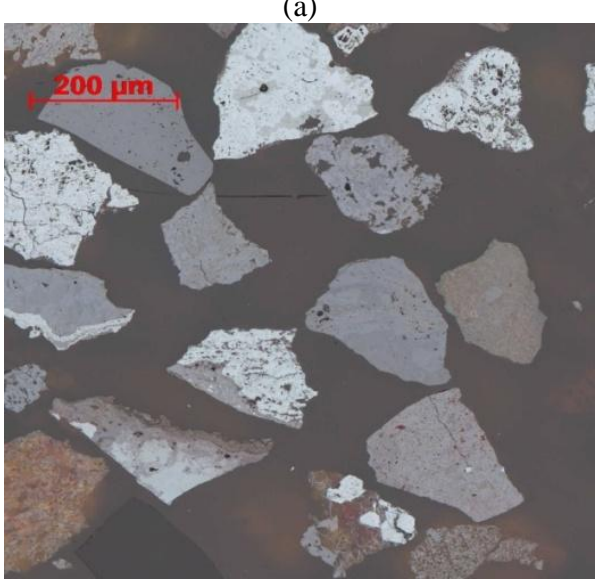

(c)

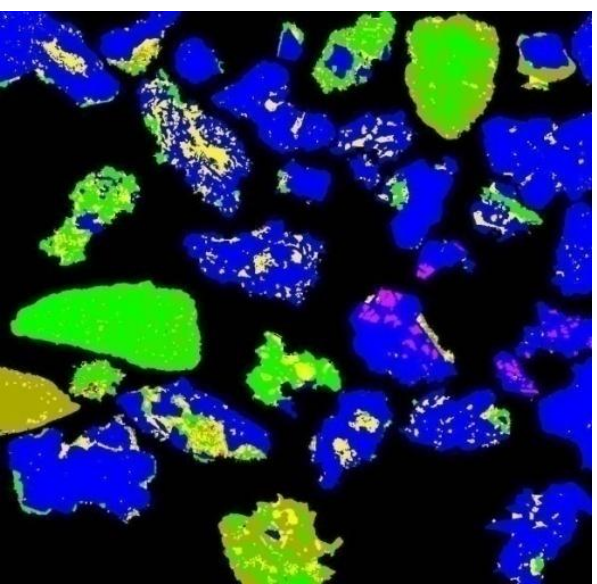

(b)

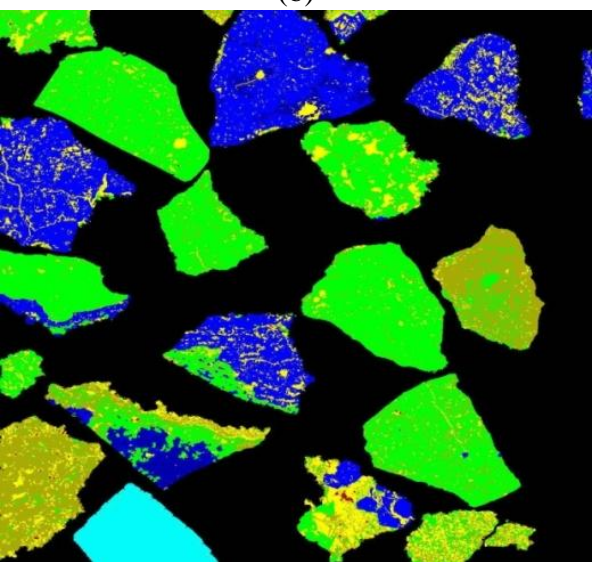

(d)

Fig. 2 Examples of optical image analysis of iron ore by Mineral 4: (a), (c) Images of samples of Brockman (with some addition of keno magnetite) and Marra Mamba fine ores; (b), (d) corresponding mineral maps obtained in Mineral 4 (magenta—keno magnetite, blue—hematite, dark blue—hydro hematite, green—vitreous goethite, olive—ochreous goethite, cyan-quartz, yellow—porosity).

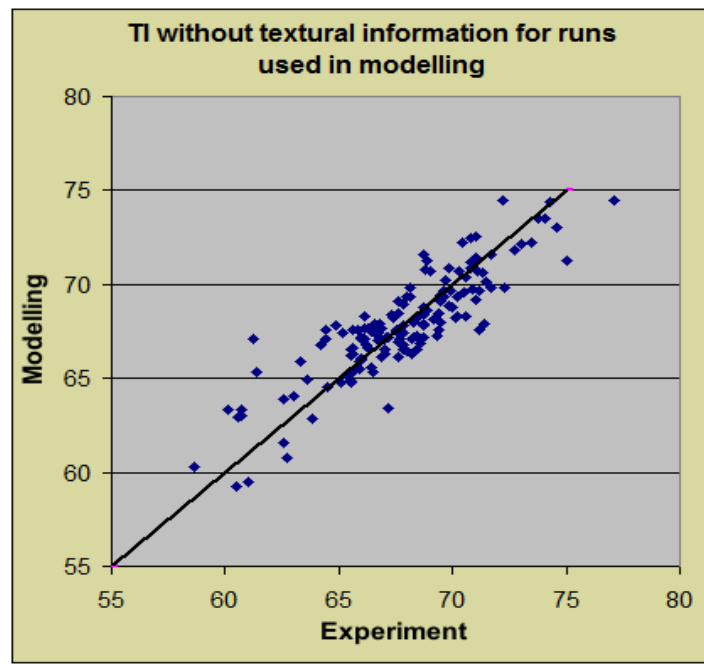

(a)

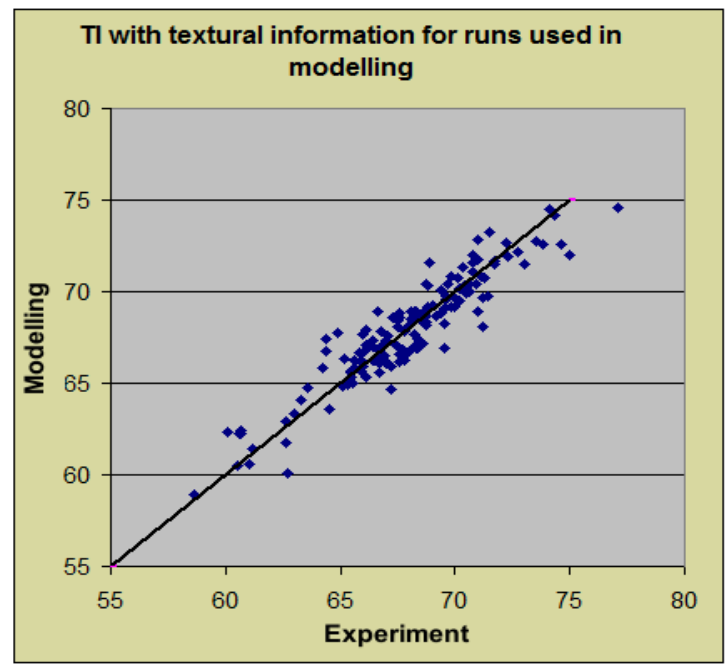

(b)

Fig. 3 Comparison of modelled and experimental tumble index values: (a) modelling without textural information; (b) modelling with textural information. 
shows a comparison between experimental results for sinter cold strength (TI) and its modelling without (a) and with (b) taking textural information into account. Based on these data, the importance of inclusion of textural information is apparent, as the modelled data became much closer to the experimental data when textural information was added. The standard deviation of the prediction (i.e. the standard deviation of the difference between experimental and predicted values) reduced from 1.53 to 1.13 . Similarly, the calculated $\mathrm{R}$-squared (the percentage of total variation in the experimental data explained by the regression model) for modelling the sinter TI (tumble index) without the inclusion of the textural information was $75.6 \%$, whereas with the inclusion of textural information it was improved to $86.9 \%$. It should be emphasized that the unexplained variation reduced almost twice from $24.4 \%$ to $13.1 \%$.

\section{Mineral 4/Recognition 4 Software}

As indicated above, the level of automation in existing SEM and OIA technologies is very high. The key difference between these existing techniques and the Mineral 4/Recognition 4 OIA package produced by CSIRO is the capability to automatically classify particles by texture.

To achieve this, the software has two main components, Mineral 4 and Recognition 4. Mineral 4

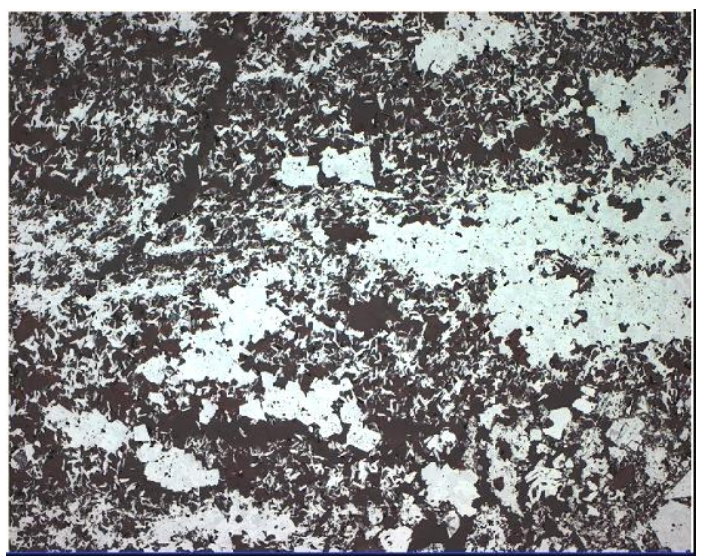

(a) is responsible for image collection, image processing, measurement of large sets of image/particle features, and output of the measurement results to Microsoft Excel and a particle/frame database. One of the key features of Mineral 4 is that it performs mineral identification and segmentation of different morphologies of the same mineral or identification of phases with similar reflectivity but different structures $[2,9]$. As an example, Fig. 4 (reproduced from Ref. [12]) demonstrates the automated segmentation of two different types of hematite-martite and micro platy hematite - that have the same reflectivity but different morphology/texture. This feature can also be used for the segmentation of different morphologies of hematite and SFCA in iron ore sinter and IMDC (inert maceral derived components) and RMDC (reactive maceral derived components) in metallurgical coke.

Recognition 4 performs the automated particle/frame texture classification, filtering, analysis and reporting. Table 1 and Table 2 give a reduced output example from a Recognition 4 based textural characteristic of an iron ore sample. Only some textural classes (altogether there were 20 classified in this ore) and major parameters are given in this table (for more details see Refs. [2, 16]). Recognition 4 calculates all major parameters for each textural group, namely: number of particles in the textural class, frequency (percentage of certain class particles by number), percentage of particles in the

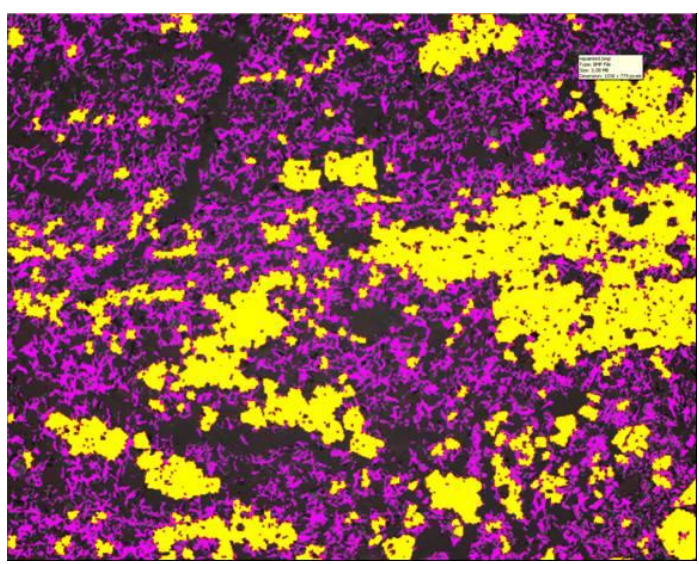

(b)

Fig. 4 Identification of martite and micro platy hematite by Mineral 4: (a) original reflected light photomicrograph; (b) mineral map (martite—yellow, micro platy hematite—magenta). 
Table 1 Example of particle textural classification output from Recognition 4 (Part 1) showing major characteristics for each textural group.

\begin{tabular}{llllllllllll}
\hline Parameters/part type & Structure & $\begin{array}{l}\text { No. of } \\
\text { part }\end{array}$ & Freq. \% & Area \% & Wt \% & $\begin{array}{l}\text { Mean } \\
\text { Fe Tot }\end{array}$ & $\begin{array}{l}\text { Mean } \\
\text { SG }\end{array}$ & $\begin{array}{l}\text { Mean } \\
\text { mineral area } \\
\left(\mu \mathrm{m}^{2}\right)\end{array}$ & $\begin{array}{l}\text { Mean shape } \\
\text { factor }\end{array}$ & Mean Elong. \\
\hline Shale-kaolinitic & Soft & 52 & 0.43 & 0.36 & 0.18 & 10.02 & 2.11 & 3,757 & 4.15 & 1.7 \\
Microplaty hematite & Medium & 1,016 & 8.39 & 8.37 & 8.05 & 69.05 & 4.15 & $4,485.3$ & 3.22 & 1.76 \\
Dense hematite & V. Hard & 5,143 & 42.45 & 43.87 & 48.93 & 69.2 & 4.81 & $4,643.1$ & 2.47 & 1.75 \\
Vitr-Ochr. goethite & Soft & 1,097 & 9.05 & 8.66 & 6.66 & 58.42 & 3.32 & $4,294.5$ & 3.08 & 1.78 \\
Martite-goethite & Hard & 1,022 & 8.44 & 8.7 & 9.34 & 67.96 & 4.64 & $4,631.6$ & 2.59 & 1.78 \\
Martite-goethite & Medium & 1,158 & 9.56 & 9.83 & 9.41 & 67.19 & 4.13 & $4,622.2$ & 3.09 & 1.73 \\
Goethite-martite & Soft & 404 & 3.33 & 3.29 & 2.56 & 61.66 & 3.36 & $4,429.8$ & 3.28 & 1.72 \\
Goethite-martite & Hard & 858 & 7.08 & 6.97 & 6.52 & 63.53 & 4.04 & $4,424.2$ & 2.92 & 1.76 \\
\hline
\end{tabular}

*abbreviations: Vitr.—vitreous; Ochr-ochreous; Freq.—frequency; Elong.—elongation.

Table 2 Example of particle textural classification output from Recognition 4 (Part 2) showing the average mineral composition and porosity of particles within each class of particle.

\begin{tabular}{|c|c|c|c|c|c|c|c|c|}
\hline Part type/mineral & $\begin{array}{l}\text { Keno-magne } \\
\text { tite }(\mathrm{Wt})\end{array}$ & $\begin{array}{l}\text { Hematite } \\
(\mathrm{Wt})\end{array}$ & $\begin{array}{l}\text { Hydro-hema } \\
\text { tite (Wt) }\end{array}$ & $\begin{array}{l}\text { Vitreous } \\
\text { goethite } \\
(\mathrm{Wt})\end{array}$ & $\begin{array}{l}\text { Ochreous } \\
\text { goethite } \\
\text { (Wt) }\end{array}$ & $\begin{array}{l}\text { Kaolinite } \\
(\mathrm{Wt})\end{array}$ & Quartz (Wt) & $\begin{array}{l}\text { Pores \% } \\
\text { (area) }\end{array}$ \\
\hline Shale-kaolinitic & 0.0876 & 1.83 & 0 & 5.31 & 9.65 & 80.12 & 2.99 & 23.08 \\
\hline Microplaty hematite & 0.0971 & 97.92 & 0.0569 & 1.26 & 0.49 & 0.13 & 0.0405 & 18.11 \\
\hline Dense hematite & 1.57 & 97.02 & 0.15 & 0.85 & 0.36 & 0.0493 & 0.0033 & 5.27 \\
\hline Vitr.-Ochr. goethite & 0.053 & 1.39 & 0.0408 & 75.65 & 21.3 & 1.53 & 0.0262 & 17.56 \\
\hline Martite-goethite & 0.93 & 84.88 & 0.59 & 11.65 & 1.86 & 0.0573 & 0.026 & 6.24 \\
\hline Martite-goethite & 0.58 & 79.19 & 0.19 & 15.86 & 3.94 & 0.22 & 0.0265 & 14.84 \\
\hline Goethite-martite & 0.37 & 34.07 & 0.18 & 45.94 & 18 & 1.41 & 0.0403 & 21.28 \\
\hline Goethite-martite & 1.18 & 38.97 & 0.61 & 53.08 & 5.97 & 0.13 & 0.0493 & 10.9 \\
\hline
\end{tabular}

class by area, weight percentage, calculated average total iron, calculated average density, average area, mean shape factor, mean elongation (Table 1). Recognition 4 also calculates variation coefficients for total iron, density and area (not shown in Table 1).

As well, the software also calculates the average mineral composition and porosity of particles within each textural class (Table 2).

For each individual textural class, the overall set of particles, or any particle subset based on criteria chosen from a wide range of options such as mineral composition, chemical composition, porosity, shape etc., much more comprehensive information can be calculated, including mineral liberation and association, mineral and density variability, dimensional characteristics, and chemical and mineral composition $[2,16]$. It should be also noted that the textural classification scheme can be changed within the software if necessary, so the same set of data can be classified differently depending on the user requirements.

\section{Characterisation of Iron Sinter}

Due to the high percentage of iron sinter in the total ferrous burden (70-85\%), sinter quality is a key element for stable blast furnace operation. Correct characterisation of iron ore sinter mineralogy and porosity is therefore very important for optimisation of the sintering process and understanding sinter quality in terms of mechanical strength and reducibility $[17,18]$.

Sinter phase characterisation is usually manually performed by an experienced mineralogist (point counting) using a microscope. Automatic identification of different morphologies of the same mineral by standard automated routines of OIA or SEM is not possible due to the fact that different morphologies of the same mineral have similar reflectivity and chemical composition. The examples below show that Mineral 
4/Recognition 4 successfully performs this task automatically using a textural identification procedure. This removes the subjectivity associated with manual point counting by eliminating opinion-based error and significantly increases productivity and the quality of data.

\subsection{Example 1: Classification of Primary versus} Secondary Hematite

During characterisation of sinter mineralogy, mineralogists/petrologists often distinguish different morphologies of the same mineral. For example, it is critical to understand how much hematitic nuclei are left unreacted after the sinter melt resolidifies which affects the sinter strength [14]. Hematite not reacted during sintering is called "primary hematite" and should be distinguished from "secondary hematite" derived from melt (see Fig. 5 (reproduced from Ref. [17])).

\subsection{Example 2: Characterisation of SFCA} Morphological Types

Another important mineral with different morphologies present in sinter is SFCA (silico-ferrite
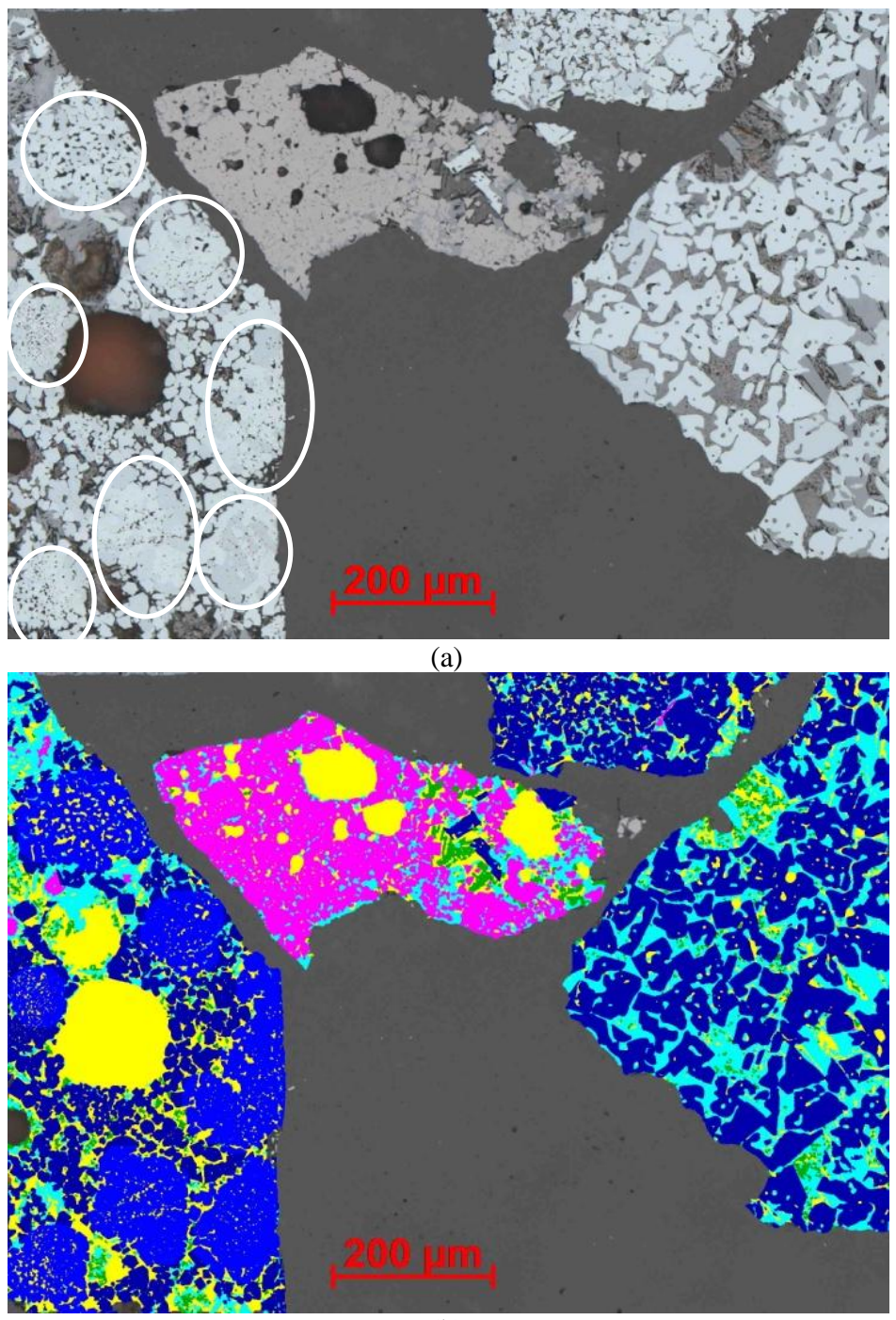

(b)

Fig. 5 (a) Typical photomicrograph of crushed sinter, and, (b) mineral map obtained during automated image analysis: primary hematite — light blue, secondary hematite—dark blue, magnetite—magenta, Fibrous SFCA—light green, Columnar SFCA — cyan, glass - dark green, porosity and epoxy within particles—yellow. In Fig. 5a, primary hematite is indicated by the circled regions. 


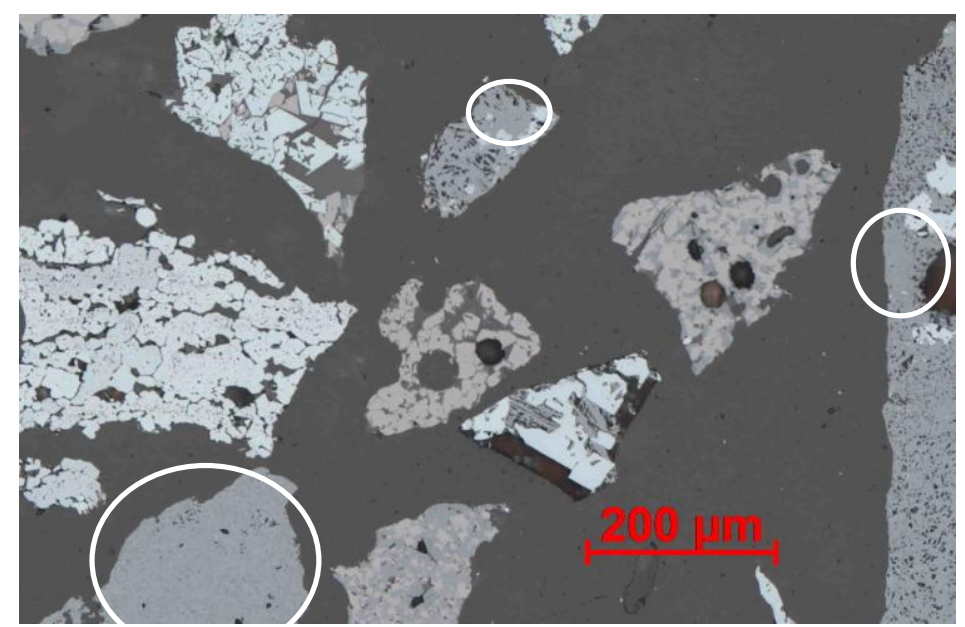

(a)

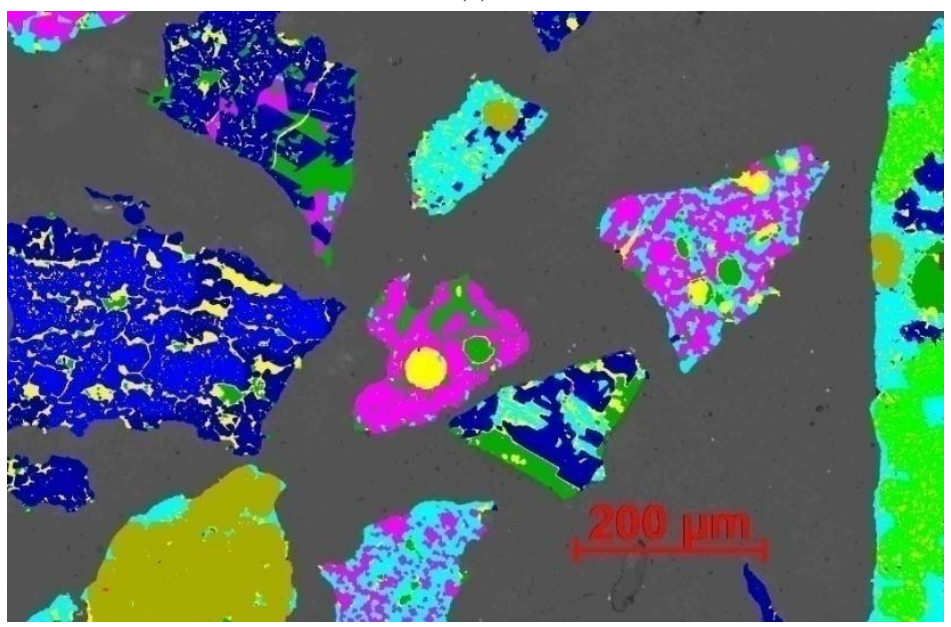

(b)

Fig. 6 (a) Photomicrograph of crushed sinter showing dense SFCA (indicated by the circled regions); (b) mineral map obtained during automated image analysis: primary hematite—light blue, secondary hematite—dark blue, magnetite—magenta, Fibrous SFCA—light green, Columnar SFCA—cyan, Dense SFCA—olive, glass+quartz—dark green, porosity and epoxy within particles-yellow.

of calcium and aluminium). Different researchers identify many different types of SFCA [19, 20] with the major types of SFCA identified by sinter petrologists as being: SFCA-I (fibrous), dense SFCA and columnar SFCA [21]. While SFCA-I has an easily recognisable micro-porous texture (see Fig. 6 (reproduced from Ref. [17])), dense SFCA has SFCA crystals inter-grown with almost no porosity (also shown in Fig. 6). Columnar SFCA most often has a prismatic morphology.

It should also be noted that, together with different morphologies of hematite and SFCA, the software identifies porosity and other minerals or phases present in sinter such as magnetite, magnesio-ferrite, glass, larnite, unreacted fluxes etc. A recent comparison between manual point counting and automated characterisation [21] revealed that manual point counting tends to underestimate minor phases present as fine inclusions. It also tends to underestimate glass due to the fact that glass reflectivity is very close to the reflectivity of epoxy making it impossible for the human eye to make a reliable distinction between them. However modern OIA, which has more than 16,000 grades in each colour channel, reliably segments glass from epoxy (see Fig. 6). The work of Hapugoda et al. [21] also showed that manual point counting tends to 
overestimate microporous phases because it does not take the presence of microporosity into account.

\section{Coke Characterisation}

Metallurgical coke is a critical component of the blast furnace burden. It performs several important functions, such as provision of reducing gases, fuel for supporting the necessary temperature, and provides a source of carbon in order for the hot metal to have certain properties [22]. Furthermore, it supports the burden to ensure good permeability for the transmission of reducing gasses and liquid phase drainage. To fulfil these tasks, coke is required to meet certain standards related to strength and reactivity.

It is accepted that coke strength is determined by its structure [23-28] in which three major components define the strength:

- Coke porosity. Patrick and Walker [27] showed that coke tensile strength has a negative correlation with pore size and elongation. Kubota et al. [23] also demonstrated the existence of a strong correlation between the total pore area and the total perimeter of low roundness pores and coke strength (as measured by $\mathrm{DI}^{150}{ }_{6}$ ).

- Coke matrix. Average pore wall thickness was correlated with coke strength by several researchers $[22,25,27]$.

- Proportion of IMDC (inert maceral derived components). Kubota et al. [24] reported that coke strength depends significantly on IMDC particle size and the presence of cracks accompanying IMDC.

The first two components were measured automatically by optical image analysis techniques during earlier research [22, 23, 25-27]. However the IMDC measurements had to be performed manually till recently [24]. The reason for this was that IMDC and RMDC (reactive maceral derived components) have highly overlapping ranges of reflectivity and cannot be segmented through simple thresholding techniques.

Coke petrologists can easily distinguish between
IMDC and RMDC based on the difference in their structure. This difference was taken into account in Mineral 4 by utilisation of the textural identification procedure that allowed automated identification of IMDC, RMDC and porosity (see Fig. 7 (reproduced from Ref. [28])). The correlation coefficient between manual point counting and automated IMDC identification in the study was 0.98 . The software initially segments the coke matrix from porosity using thresholding, and after that it uses three different structural identification methods to segment IMDC from RMDC [28, 29]. The results of these three methods are combined to obtain the final identification of IMDC. These methods include:

- Bulk identification of IMDC, which is based on the appearance of non-reacted IMDC as a large nucleus surrounded by RMDC having a reacted "network" structure;

- Fine porosity IMDC identification which segments areas with a high level of microporosity;

- "Washed out" areas method, which is based on observations of areas in some polished blocks which have lower reflectivity than the overall coke matrix. They are assumed to be areas of IMDC partially destroyed during epoxy block preparation due to insufficient resin impregnation of the sample, or IMDC with high micro/nano-porosity (see IMDC in the white ellipse Fig. 7).

To demonstrate the textural identification capabilities of Mineral 4, additional examples of "Fine porosity IMDC identification" are provided below. Fig. 8 illustrates different stages of this method applied to the image of a coke sample shown in Fig. 8a. In the first stage, the overall porosity map and map of the solid part of coke matrix are segmented by thresholding.

The fine porosity is then extracted from the whole porosity map (Fig. 8b). In the next stage, areas (clusters) of concentrated fine porosity are identified (Fig. 8c). Finally, by masking with the map of the solid part of the coke, microporous IMDC areas are determined (Fig. $8 d)$. 


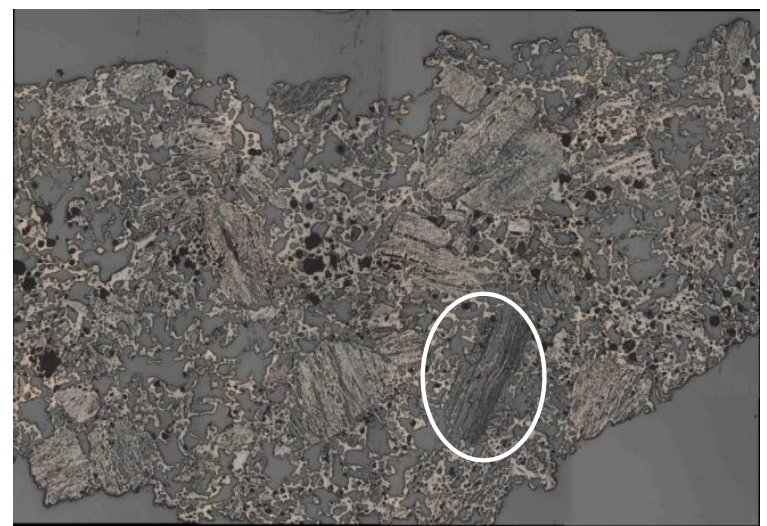

(a)

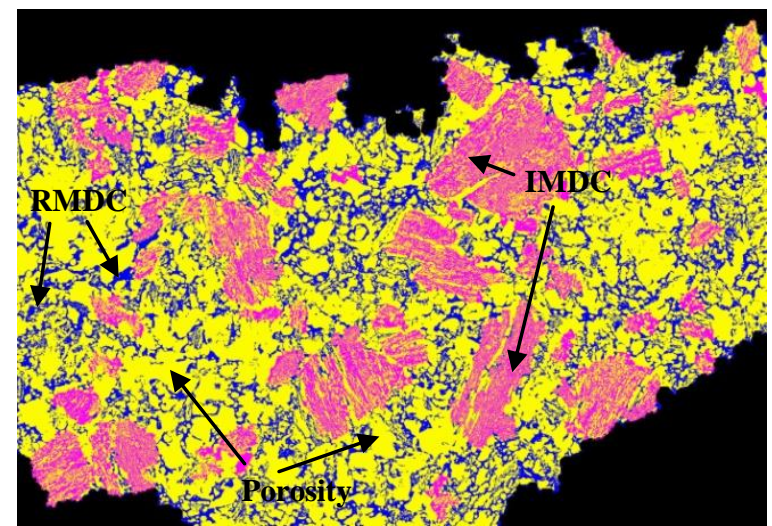

(b)

Fig. 7 Automated identification of IMDC: (a) original reflected light photomicrograph of a coke sample ("Washed out" IMDC circled); (b) automatically mapped IMDC (magenta), RMDC (blue) and porosity (yellow).

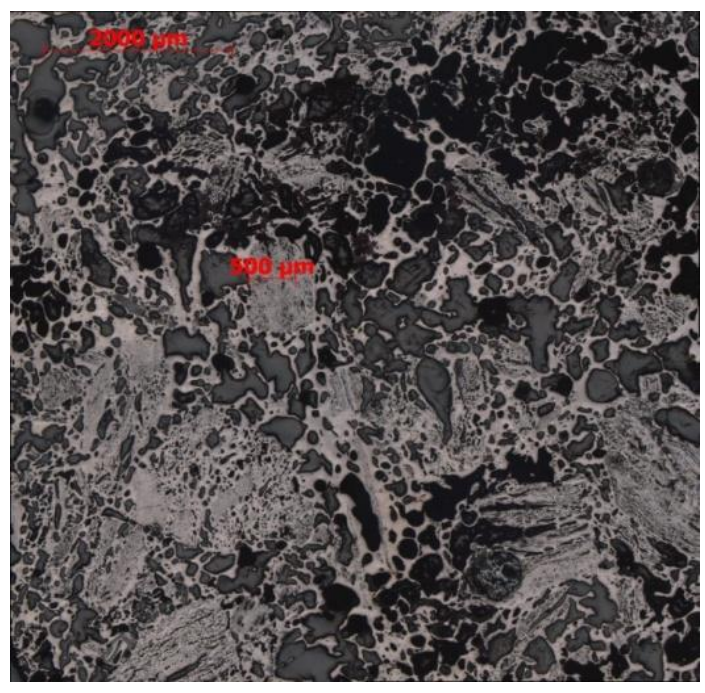

(a)

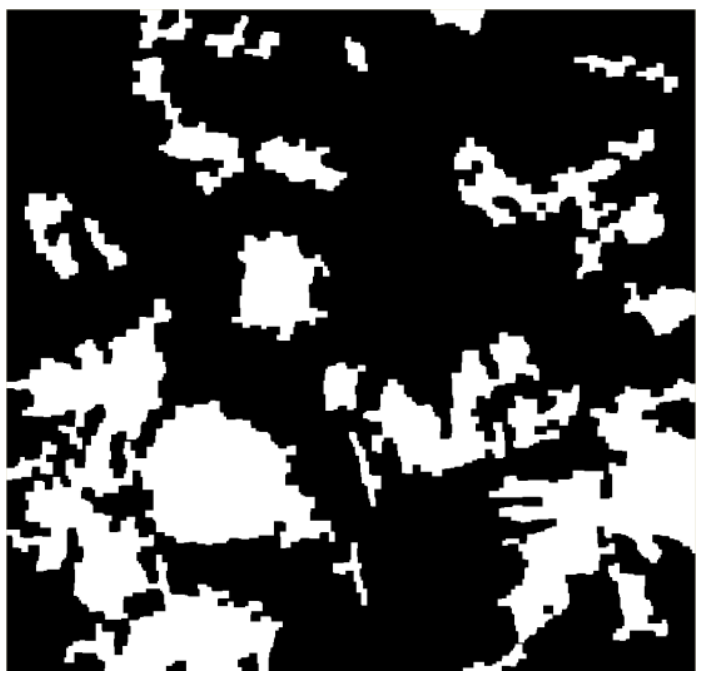

(c)

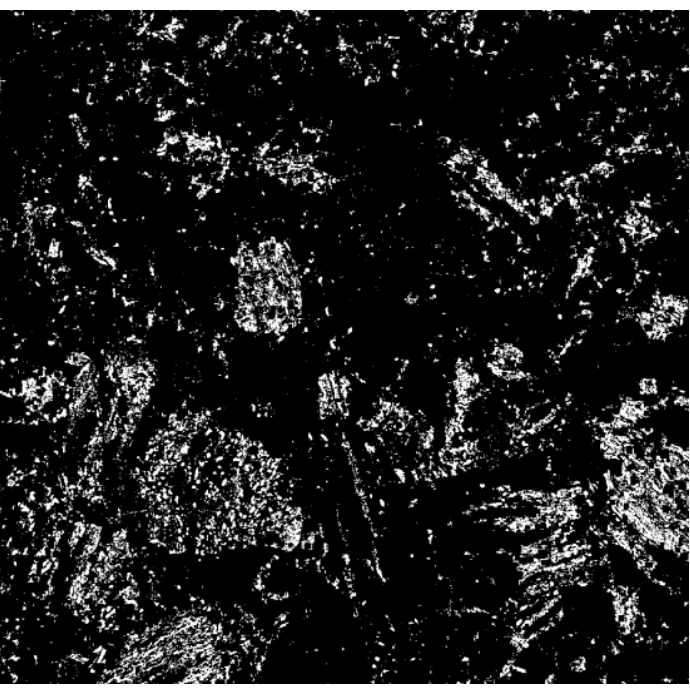

(b)

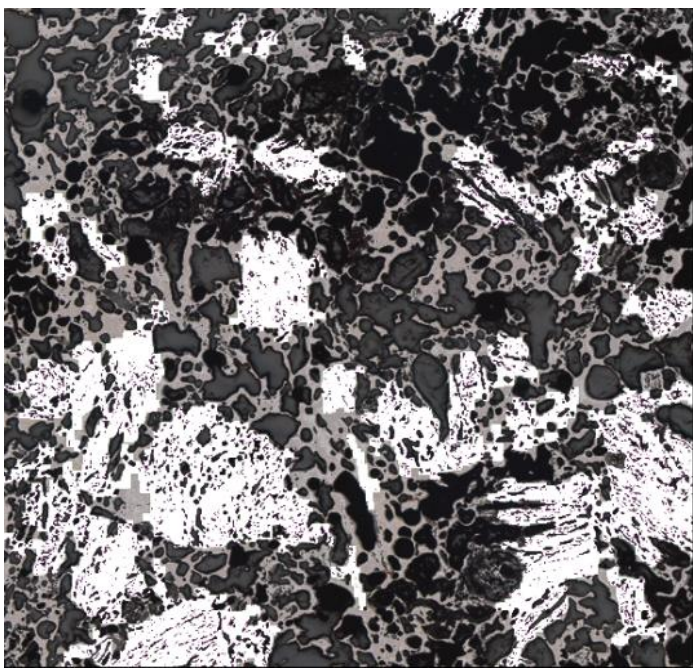

(d)

Fig. 8 Photomicrograph of coke (a) intermediate maps in "Fine Porosity IMDC identification", (b) fine porosity map, (c) areas of concentrated fine porosity, and (d) final map of microporous parts of IMDC (white). 
The characterisation of IMDC also includes calculation of the IMDC size distribution for the whole group, for each size fraction, or for each IMDC area separately (if needed). Such calculations include the average area of IMDC, the convex area of IMDC region (area of convex shell), the perimeter, the ratio of ferets (elongation), the equivalent circle diameter, convexity, concavity, solidity (see Ref. [22]), shape factor, smoothness of IMDC boundary and boundary length. Porosity characterisation also includes distributions with similar descriptors. Donskoi et al. [28] showed that to properly reflect the effect of large objects (pores, IMDC), and to reduce the effect of small objects, averaging of characteristics should be performed using weighting by object area. Mineral 4 calculates averages for each characteristics both by number and by area.

One of the weakest areas in coke is the boundary area between the IMDC and RMDC regions, which often has elongated pores capable of promoting cracking under mechanical stress. Mineral 4 segments this boundary (Fig. 9) and calculates parameters describing the walls that connect IMDC and RMDC as well as the voids between them contained within the boundary layer. As a result, quantitative descriptors of IMDC-RMDC connectivity are provided which can be correlated with coke strength qualifiers. Such parameters include average thickness of RMDC walls connected to IMDC in the boundary area, abundance of RMDC walls in the boundary area of IMDC, average distance between RMDC walls in the IMDC boundary area and maximal distance between RMDC walls in the IMDC boundary area.

The capabilities of the software also allow measurement of many other important parameters characterising coke structure, such as, average wall thickness considering the whole coke matrix, average wall thickness considering only RMDC part of the matrix, or average wall thickness considering only connections between relatively large agglomerations in

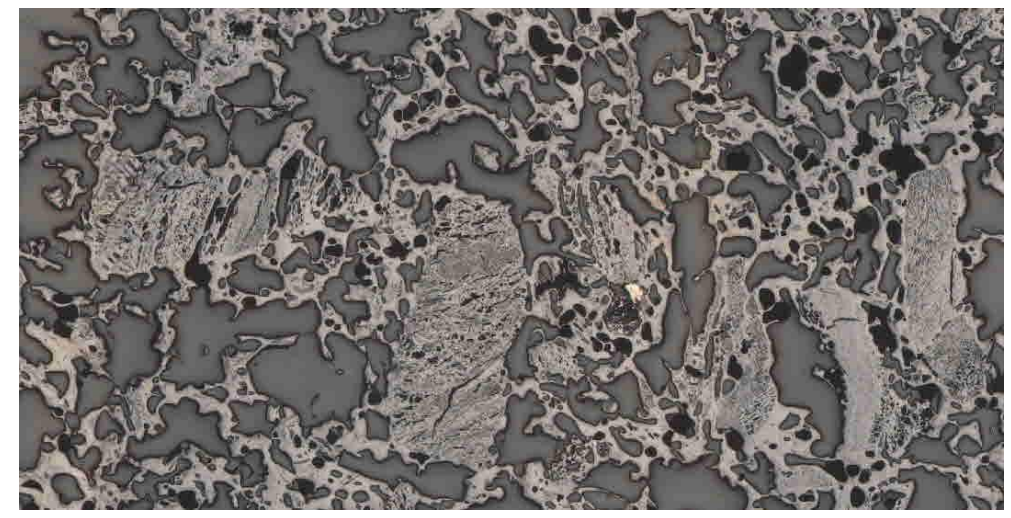

(a)

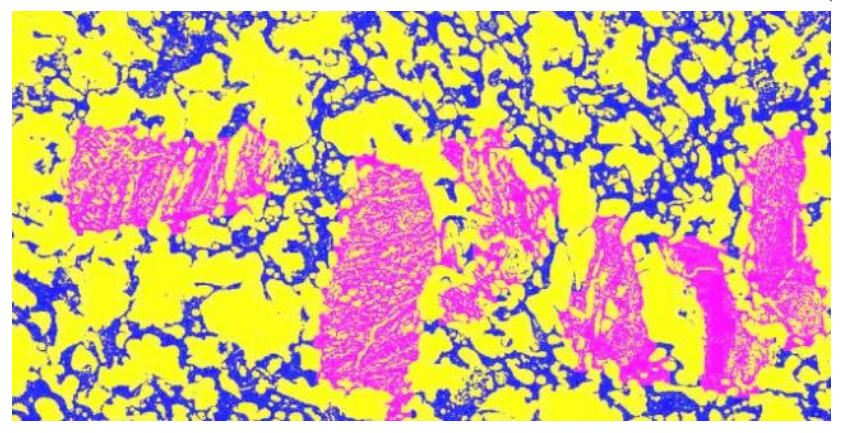

(b)

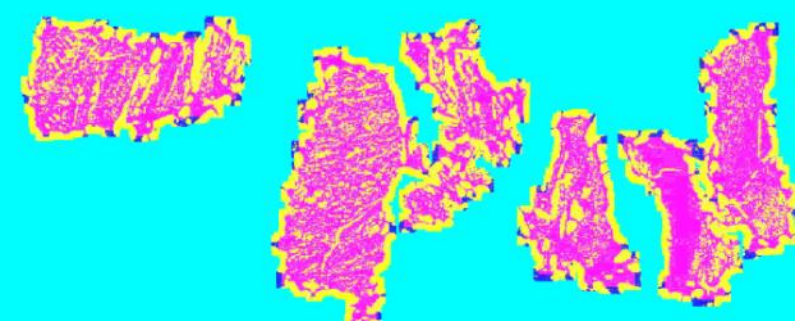

(c)

Fig. 9 Segmentation of IMDC boundary layers: (a) initial image, (b) identified IMDCs and RMDCs, and (c) IMDCs only with allocated boundary layers, (IMDC—magenta, RMDC—blue and porosity—yellow). 
the coke matrix (represented by large areas of IMDC) and large coagulations of RMDC. The capability of measuring the presence of one phase within another allows the measurement of average recordable porosity within a particular phase such as IMDC or RMDC.

A discussion of the complete range of capabilities of Mineral 4/Recognition 4 in coke structure characterisation is beyond the scope of this paper. More details can be found in the ACARP (Australian Coal Association Research Program) report "Optical image analysis of coke structure and the effects of structural parameters on coke strength" [29].

\section{Lump and Pellet Characterisation}

The Mineral 4/Recognition 4 software also has the capability to analyse the structures of coarse particles such as pellets or large lumps of ore or sinter. As the analysis of minerology, porosity and texture is performed under a microscope, significant magnification is required for proper identification of mineralogy, porosity and texture, and so individual images cover only a small area of the sample. However, for effective characterisation of such materials, it is necessary to analyse large areas, such as the whole section of an indurated pellet, so that the size of the total image, combined from the individual images, can be hard to handle. Another issue encountered when conducting characterisation of these larger material types is that the individual image should be small to ensure there are enough points in the spatial distribution of the studied characteristic, but at the same time it should be large enough to be capable of averaging out local irregularities.

To solve these problems, while maintaining the capability to combine individual images into large Mosai X images, Mineral 4 further combines Mosai X images into "panorama" images. All mosaic images can be separately processed, and the results of such processing presented as tables and graphs.

Fig. 10a shows a "panorama" image of an indurated pellet manufactured from a magnetite concentrate. Due to the oxidising conditions during induration, thin rims of hematite can be seen around some magnetite grains (Fig. 10c). Fig. 11 demonstrates Mineral 4 mineral mapping of the images in Fig. 10a. In these images magnetite, is represented with the magenta colour, hematite with blue, porosity with yellow and small hardly recognisable binder areas with green.

Fig. 12 demonstrates spatial distributions of magnetite, hematite and porosity in the pellet from Fig. 10a. These smooth spatial distributions are obtained by applying a moving average procedure. Mineral 4 allows adjustments of parameters determining resolution of such images. Spatial distributions in Fig. 12 show that while there is some symmetry along one direction of the pellet, another direction is significantly asymmetrical, possibly due to asymmetrical processing (e.g. heating, asymmetrical gas distribution etc.) during induration. The image clearly identifies an outer ring of hematitic material caused by the oxidation of the magnetite as the pellet was indurated. Such information can assist the induration plant operator in determining if the firing cycle was sufficiently rigorous. The abundances can also be output to Excel, both as the whole distribution matrix for each mineral and data averaged for images that are at the same distance from the centre, and used for further calculations or visualisation as in the example in Fig. 13.

\section{Conclusions}

Particles with the same mineralogy but different texture can behave very differently during downstream processing. Consequently, access to objective textural information for iron ores is very valuable for understanding and predicting their processing behaviour. Optical image analysis, in comparison with SEM methods, can better identify micro-porosity and segment different iron oxides and oxyhydroxides. The superior capability of optical image analysis together with the specific capabilities of the Mineral 4/Recognition 4 system to perform identification of different textures, non-opaque minerals and provide comprehensive 

Sinter and Coke Characterization

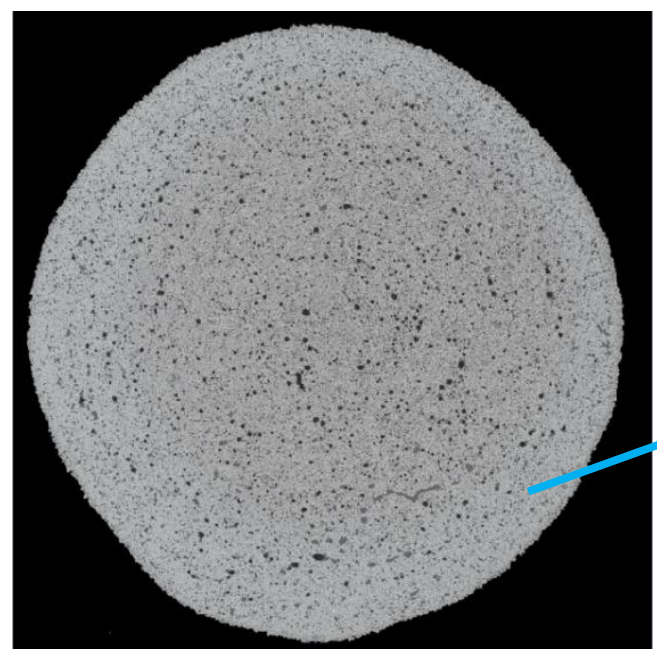

(a)

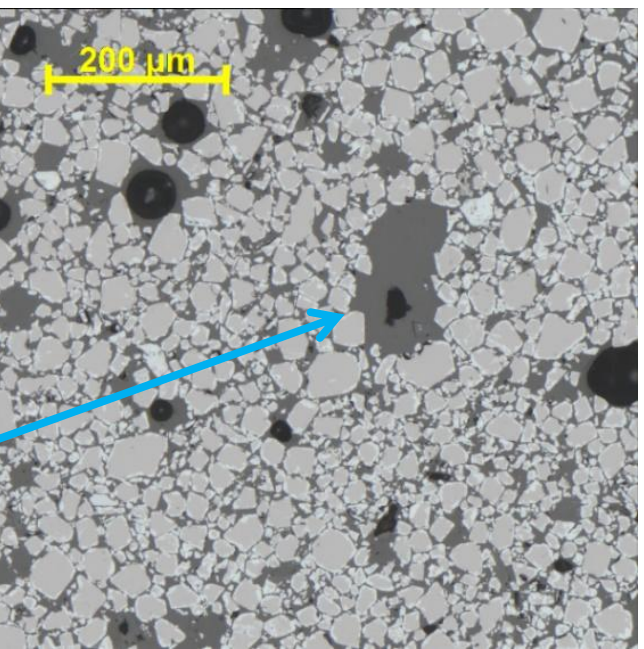

(b)

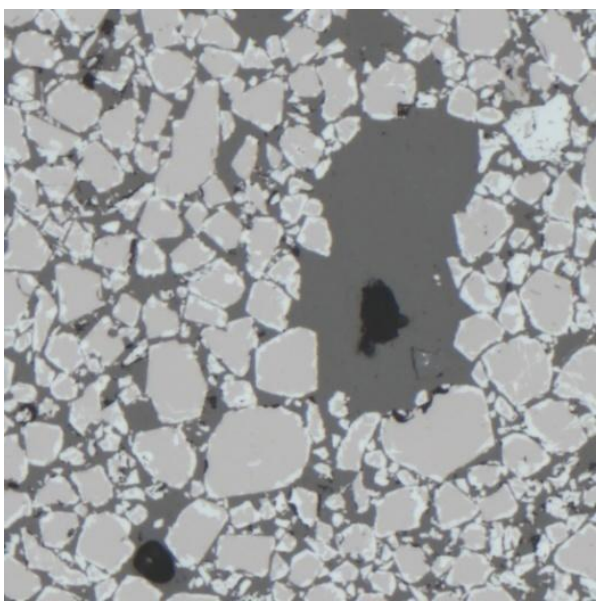

(c)

Fig. 10 (a) Whole pellet image $(\varnothing 12.7 \mathrm{~mm}$ ) - a combination of $18 \times 18$ Mosai $\mathrm{X}$ images where each image is a combination of $2 \times 2$ elementary images; (b) Mosai X image from the pellet area marked with arrow (magnetite-pink, hematite-white, porosity-dark); (c) a closer view of image (b).

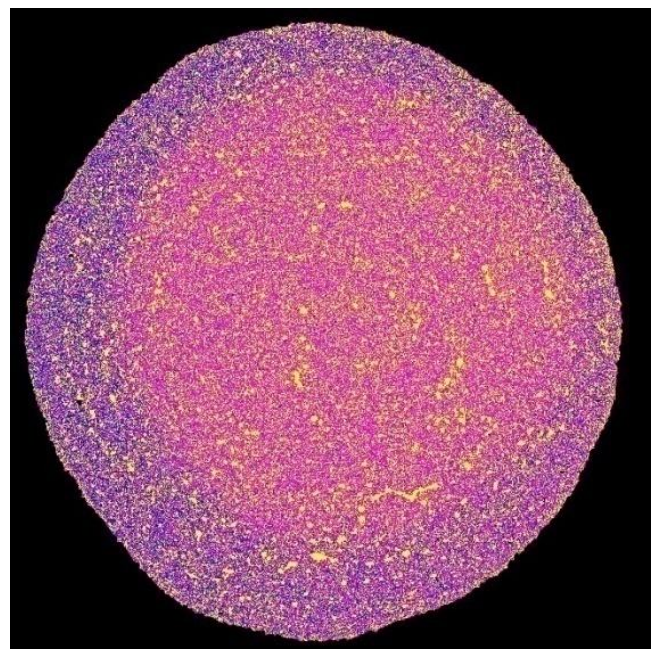

(a)

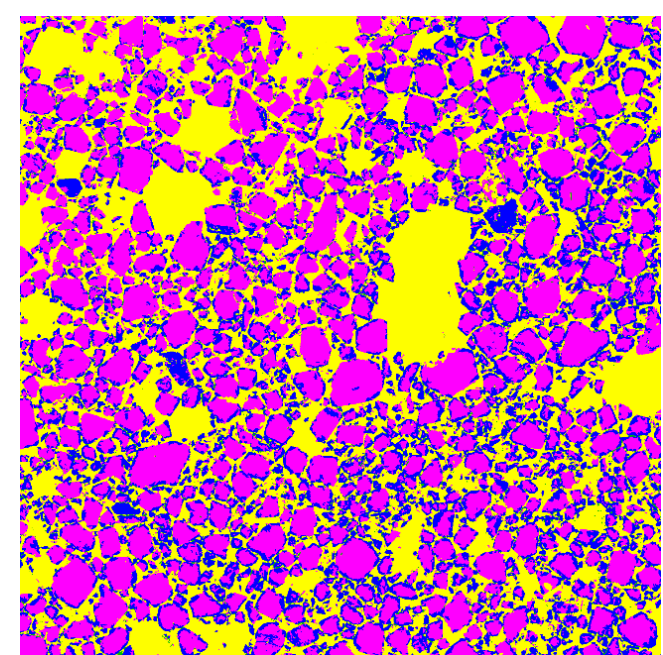

(b)

Fig. 11 (a) Mineral mapping of the pellet in Fig. 10a; (b) mineral mapping of Mosai X image from Fig. $10 b$. 

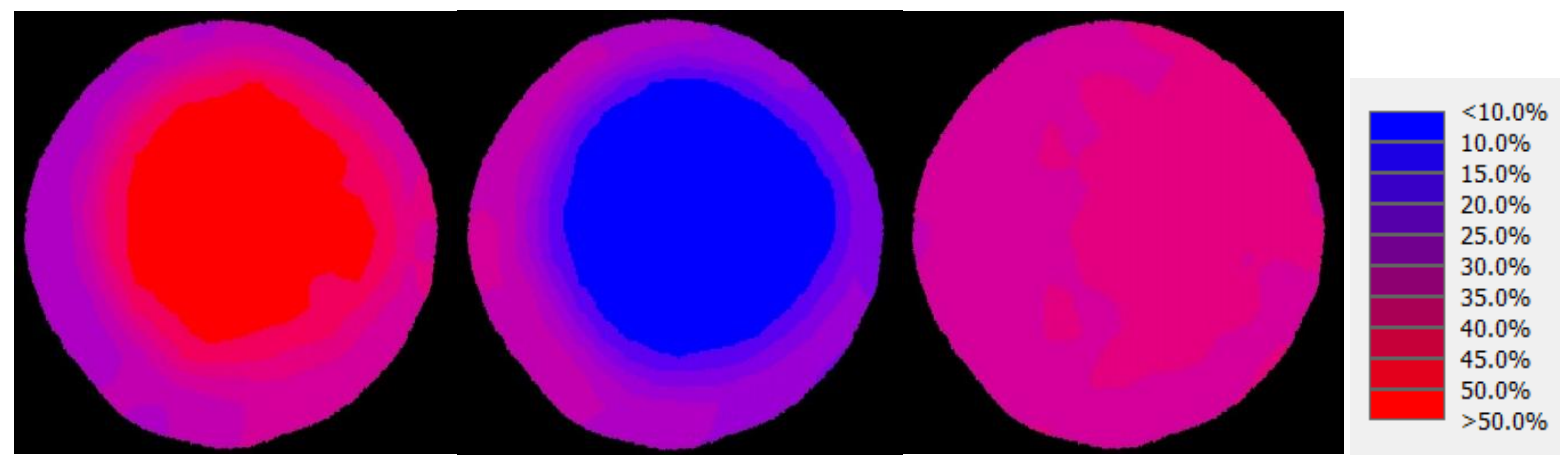

Fig. 12 Spatial distributions of magnetite (left), hematite (centre) and porosity (right) of the pellet on Fig. 10a. Abundances calculated for each of the $18 \times 18$ Mosai $\mathrm{X}$ images, then an adaptive moving average is used to smooth the visualisation.

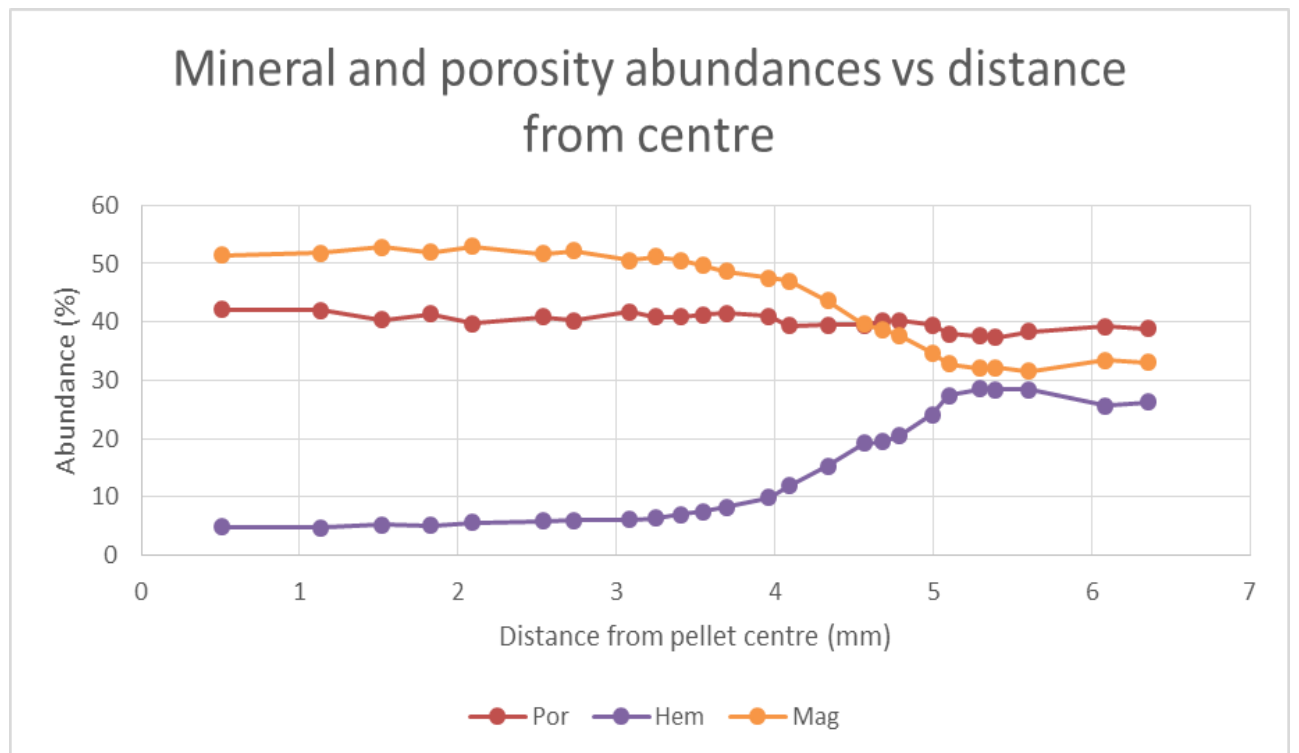

Fig. 13 Graph of average hematite (Hem), magnetite (Mag) and porosity (Por) abundances as a function of distance from pellet centre.

image analysis allow automatic textural classification of particle sections. The resulting data output files provide comprehensive textural, dimensional, liberation, association and other capacious information about each texture class, group of particles or any sub-group defined by a flexible choice of criteria.

Textural identification and multi-thresholding procedures, which are the key features in Mineral 4 for mineral identification, allow identification of different morphologies of the same mineral. This is very important for iron sinter characterisation where Mineral 4 is capable of automatically segmenting primary hematite from secondary hematite, and distinguishing between the three different types of SFCA. Such automated identification removes the subjectivity that may be present during manual mineral identification by mineralogist/petrologist. Mineral 4/Recognition 4 also has significant advantages in comparison with manual point counting in identification of micro-porosity, glass, and characterisation of microporous phases.

Textural identification also allows for differentiation of IMDC and RMDC in cokes. This allows for comprehensive characterisation of IMDC, RMDC and porosity distributions, with all data calculated with averaging by number and by area. Structural characterisation of the coke matrix also gives additional comprehensive information, including description of the boundaries between IMDC and RMDC, the average wall thickness and the porosity of IMDC. 
Characterisation of relatively large objects such as pellets and lumps of iron ore and sinter is also very important for research related to the iron making industry. By combining Mosai X images into large "panorama" images, Mineral 4/Recognition 4 provides the opportunity to study such macro-objects as a whole.

In conclusion, this article clearly demonstrates that the unique features and capabilities created in CSIRO's Mineral 4/Recognition 4 software allow comprehensive and, in some cases, unique characterisation of important features relevant to iron making researchers and industry plant operators.

\section{Acknowledgments}

The authors wish to thank CSIRO Mineral Resources and ACARP (the Australian Coal Association Research Program) for funding this research and development. The authors also wish to thank the staff of the CSIRO Carbon Steel Futures group for valuable suggestions and help during this work. We would also like to express our acknowledgments to our internal reviewers Mark Pownceby and Mike Peterson for their useful corrections and comments.

\section{References}

[1] Ramanaidou, E., Wells, M., Belton, D., Verrall, M., and Ryan, C. 2008. "Mineralogical and Microchemical Methods for the Characterization of High-Grade Banded Iron Formation-Derived Iron Ore." In Banded Iron Formation-Related High-Grade Iron Ore. Vol. 15. Society for Economic Geologists.

[2] Donskoi, E., Poliakov, A., Manuel, J., Peterson, M., and Hapugoda, S. 2015. "Novel Developments in Optical Image Analysis for Iron Ore, Sinter and Coke Characterisation." Applied Earth Science (Transactions of the Institutions of Mining and Metallurgy: Section B) 124 (4): 227-44.

[3] Donskoi, E., Poliakov, A., Holmes, R., Suthers, S., Ware, N., Manuel, J., and Clout, J. 2016. "Iron Ore Textural Information Is the Key for Prediction of Downstream Process Performance." Minerals Engineering 86 (February): 10-23.

[4] Iglesias, J. C. A., Gomes, O. D. F. M., and Pasiornik, S. 2011. "Automatic Recognition of Hematite Grains under Polarized Reflected Light Microscopy through Image Analysis." Minerals Engineering 24 (12): 1264-70.

[5] Gottlieb, P., Wilkie, G., Sutherland, D., Ho-Tun, E., Suthers, S., Perera, K., Jenkins, B., Spencer, S., Butcher, A., and Rayner, J. 2000. "Using Quantitative Electron Microscopy for Process Mineralogy Applications." JOM Journal of the Minerals, Metals \& Materials Society 52 (4): 24-5.

[6] Gu, Y., and Guerney, P. 2000. "Improving Flotation Plant Performance with the JKMRC/Philips Mineral Liberation Analyser." Present at International Conference Minerals Engineering, Falmouth, Cornwall.

[7] Maddren, J., Ly, C. V., Suthers, S. P., Butcher, A. R., Trudu, A. G., and Botha, P. W. S. K. 2007. "A New Approach to Ore Characterisation Using Automated Quantitative Mineral Analysis.” In Iron Ore-Proceedings-CD-ROM Edition, The Australasian Institute of Mining and Metallurgy, 131-2.

[8] Pirard, E., Lebichot, S., and Krier, W. 2007. "Particle Texture Analysis Using Polarized Light Imaging and Grey Level Intercepts." International Journal of Mineral Processing 84 (1-4): 299-309.

[9] Donskoi, E., Poliakov, A., and Manuel, J. R. 2015. "Automated Optical Image Analysis of Natural and Sintered Iron Ore." In Iron Ore: Mineralogy, Processing and Environmental Issues, edited by Lu, L. Amesterdam: Elsevier, 101-59.

[10] Gomes, O. D. M., and Paciornik, S. 2008. “ Iron Ore Quantitative Characterization through Reflected Light-Scanning Electron Co-site Microscopy." In Proceedings of the 9th International Congress for Applied Mineralogy, 699-702.

[11] Gomes, O. D. M., and Paciornik, S. 2008. "RLM-SEM Co-site Microscopy Applied to Iron Ore Characterization." In Annals-2nd International Symposium on Iron Ore, 218-24.

[12] Donskoi, E., Manuel, J., Austin, P., Poliakov, A., Peterson, M., and Hapugoda, S. 2014. "Comparative Study of Iron Ore Characterisation Using a Scanning Electron Microscope and Optical Image Analysis." Applied Earth Science IMM Transactions section B 122 (4): 217-29.

[13] Lund, C., Lamberg, P., and Lindberg, T. 2015. "Development of a Geometallurgical Framework to Quantify Mineral Textures for Process Prediction." Minerals Engineering 82 (May): 61-77.

[14] Donskoi, E., Manuel, J. R., Clout, J. M. F., and Zhang, Y. 2007. "Mathematical Modelling and Optimization of Iron Ore Sinter Properties." Israel Journal of Chemistry (Online) 47 (3-4): 373-9.

[15] Donskoi, E., Manuel, J. R., Lu, L., Holmes, R. J., Poliakov, A., and Raynlyn, T. 2009. "Advances in Mathematical 
Modelling of Sintering Performance of Iron Ore Fines." In Proceedings of the Iron Ore Conference, 341-9.

[16] Donskoi, E., Suthers, S. P., Fradd, S. B., Young, J. M., Campbell, J. J., Raynlyn, T. D., and Clout, J. M. F. 2007. "Utilization of Optical Image Analysis and Automatic Texture Classification for Iron Ore Particle Characterisation.” Minerals Engineering 20 (5): 461-71.

[17] Donskoi, E., Hapugoda, S., Lu, L., Poliakov, A., Peterson, M., and Haileslassie, A. 2015. "Advances in Optical Image Analysis of Iron Ore Sinter." In Proceedings of the Iron Ore, 543-8.

[18] Pimenta, H. P., and Seshadri, V. 2002. "Characterisation of Structure of Iron Ore Sinter and Its Behaviour during Reduction at Low Temperatures." Ironmaking and Steelmaking 29 (3): 169-74.

[19] Sasaki, M., and Hida, Y. 1982. "Considerations on the Properties of Sinter from the Point of Sintering Reaction." Tetsu-to-Hagane 68 (6): 563-71.

[20] Ahsan, S. N., Mukherjee, T., and Whiteman, J. A. 1983. "Structure of Fluxed Sinter." Ironmaking and Steelmaking 10 (2): 54-64.

[21] Hapugoda, S., Lu L., Donskoi, E., and Manuel, J. 2016. "Mineralogical Quantification of Iron Ore Sinter." Mineral Processing and Extractive Metallurgy 125 (3): 156-64.

[22] Andriopoulos, N., Loo, C. E., Dukino, R., and McGuire, S.
J. 2003. "Micro-properties of Australian Coking Coals." ISIJ International 43 (10):1528-37.

[23] Kubota, Y., Nomura, S., Arima, T., and Kato, K. 2011. "Quantitative Evaluation of Relationship between Coke Strength and Pore Structure." ISIJ International 51 (11): 1800-8.

[24] Kubota, Y., Nomura, S., Arima, T., and Kato, K. 2008. "Effects of Coal Inertinite Size on Coke Strength." ISIJ International 48 (5): 563-71.

[25] Grant, M. J. K., Chaklader, A. C. D., and Price, J. T. 1991. "Factors Affecting the Strength of Blast Furnace Coke." Fuel 70 (2): 181-8.

[26] Sato, H., Patrick, J. W., and Walker, A. 1998. "Effect of Coal Properties and Porous Structure on Tensile Strength of Metallurgical Coke.” Fuel 77 (11): 1203-8.

[27] Patrick, J. W., and Walker, A. 1989. "Macroporosity in Cokes: Its Significance, Measurement, and Control." Carbon 27 (1): 117-23.

[28] Donskoi, E., Poliakov, A., and Lu, L. 2013. "Advances in Optical Image Analysis of Coke Structure." In Proceedings of the 10th Australian Coal Science Conference, 17.

[29] Donskoi, E., Poliakov, A., and Mahoney, M. 2015. Optical Image Analysis of Coke Structure and the Effects of Structural Parameters on Coke Strength. ACARP project C23051, CSIRO report EP153398, 1-118. 